Relations industrielles

Industrial Relations

\title{
Grèves et lock-out au Québec - 1978, Rapport annuel, Québec, Services des études en relations et conditions de travail, ministère du Travail et de la Main-d'oeuvre, juillet 1979, 69 pp.
}

\section{Jacques Mercier}

Volume 35, numéro 1, 1980

URI : https://id.erudit.org/iderudit/029047ar

DOI : https://doi.org/10.7202/029047ar

Aller au sommaire du numéro

Éditeur(s)

Département des relations industrielles de l'Université Laval

ISSN

0034-379X (imprimé)

1703-8138 (numérique)

Découvrir la revue

Citer ce compte rendu

Mercier, J. (1980). Compte rendu de [Grèves et lock-out au Québec - 1978,

Rapport annuel, Québec, Services des études en relations et conditions de

travail, ministère du Travail et de la Main-d'oeuvre, juillet 1979, 69 pp.]

Relations industrielles / Industrial Relations, 35(1), 165-166.

https://doi.org/10.7202/029047ar

Tous droits réservés @ C Département des relations industrielles de l'Université Laval, 1980
Ce document est protégé par la loi sur le droit d'auteur. L'utilisation des services d’Érudit (y compris la reproduction) est assujettie à sa politique d'utilisation que vous pouvez consulter en ligne.

https://apropos.erudit.org/fr/usagers/politique-dutilisation/ 
La grande dépression, cependant, n'a pas été marquée qué par une élévation sans précédent des taux de chômage. Celui-ci était une conséquence plus qu'une cause. Au fond, c'est le déclin massif des valeurs monétaires et l'effondrement de la demande et de la production qui l'ont provoqué. C'est pourquoi il fallait, non seulement en chercher la guérison par des mesures d'aide aux sans-travail sous quelque forme que ce soit, mais trouver les moyens de remettre la machine économique en marche.

Au départ, la guerre y a pourvu. Mais il ne faut pas négliger l'apport des économistes et, de tous ceux-ci, un nom vient immédiatement à l'esprit: John Maynard Keynes dont le célèbre ouvrage General Theory of Employment, Interest, and Money a influencé et influence encore la politique économique de bien des nations occidentales. Pour Keynes, l'épargne n'était pas nécessairement une vertu. Ce qui importait, durant les dépressions, c'était de stimuler la consommation. Pour cela, il fallait entreprendre de vastes programmes de travaux publics, accroître le crédit, même au risque de provoquer l'inflation. Au point de vue pratique, cette phrase d'un autre économiste traduit bien la pensée de Keynes «Si quelqu'un emploie 100,000 personnes et leur paie des gages, elles dépensent ces gages et l'argent ainsi dépensé emploie d'autres hommes». Keynes visait donc à la réalisation du plein emploi. Il ne devait pas atteindre le but qu'il cherchait, parce qu'il y a bien d'autres formes de chômage que le chômage cyclique, mais sa théorie, une fois appliquée, a permis au monde occidental de connaître, au cours des trente dernières années une ère véritable de prospérité. Ce fut le triomphe de la société d'abondance et de la société de consommation.

Mais cette période touche-t-elle à sa fin avec l'apparition du nouveau phénomène connu sous le nom de stagflation? L'auteur y consacre les deux derniers chapitres de son ouvrage. Pour lui, c'est peut-être la fin de l'âge d'or. Si le chômage est une maladie des sociétés industrielles modernes, l'inflation est aussi une de ses caractéristiques, et les rapports entre l'un et l'autre sont intimes. Les vérités que Keynes a expliquées demeurent mais «la théorie générale» n'apparaît plus être tellement générale. Ne serait-elle pas un cas spécial qui n'est applicable que dans certaines conditions et à une certaine époque. $\mathrm{La}$ nôtre n'aurait-elle pas besoin d'un nouveau Keynes, mais ce sauveur ne semble pas encore être en vue.

Ce qui précède est un résumé bien pâle et fort incomplet d'un ouvrage fortement documenté. En entreprenant une synthèse de l'histoire de certains aspects du chômage à travers les âges, l'auteur s'engageait dans une entreprise difficile. Il n'a pas raté l'objectif et c'est ce qui rend la lecture de cet ouvrage à la fois captivante et utile: captivante parce qu'on peut y découvrir comment les hommes dans des siècles et des pays différents ont réagi devant la personne privée de travail ou incapable de travailler; utile, surtout dans les derniers chapitres, parce qu'elle oblige à réfléchir aux lendemains de l'époque actuelle où il faut faire face au cercle vicieux du chômage et de l'inflation juxtaposés.

André ROY

Québec

Grèves et lock-out au Québec - 1978, Rapport annuel, Québec, Services des études en relations et conditions de travail, Ministère du Travail et de la Main-d'oeuvre, juillet 1979, $69 \mathrm{pp}$.

Il s'agit du second rapport annuel sur le sujet publié par le MTMO; le premier ainsi qu'un document méthodologique préliminaire ont déjà été recensés dans les numéros 1 et 3 du volume 33 de Relations industrielles. Comme toute jeune publication, la présente connaît et connaîtra encore certaines modifications destinées à en améliorer la qualité et le volume d'information. Rappelons que "Grèves et lockout» vise à cerner au moyen de données statistiques un type de manifestation des conflits de travail, à savoir les arrêts 
de travail. Les principaux indices (tableau II) en sont le nombre de conflits, le nombre de travailleurs touchés, le nombre de jourspersonnes perdus, la durée moyenne de jours ouvrables perdus et le pourcentage de temps de travail perdu estimé. Certains indices sont ensuite ventilés selon des caractéristiques telles le secteur d'activité, la région, l'affiliation syndicale, le mois, le type de conflits.

On a retiré cette année le tableau «conflits de travail selon les sources d'information". On retrouve par contre quatre nouveaux tableaux (VII à $X$ ). Le premier offre une désaggrégation plus poussée des conflits par secteurs d'activités tandis que les trois autres permettent de croiser entre eux les indices de nombre de conflits, nombre de travailleurs en cause et nombre de jours-personnes perdus en ventilant l'indice de référence. On apprend par exemple au tableau IX, qu'environ $50 \%$ des arrêts sont de moins de deux semaines et comptent près de $80 \%$ des travailleurs impliqués dans des grèves ou lock-out, tandis que les arrêts de plus de trois mois représentent $82 \%$ des grèves et lock-out et $2.6 \%$ des travailleurs en cause. La possibilité de ventiler ces données selon d'autres variables telles l'affiliation syndicale, les secteurs d'activité, l'autorité, le cadre du conflit, etc. ouvrent des perspectives d'analyse fort intéressantes pour cerner davantage la nature des arrêts de travail au Québec.

De fait, la possibilité d'accroître la quantité d'information sur les arrêts de travail provient de données recueillies à l'aide d'un questionnaire administré auprès de chaque employeur impliqué dans un arrêt de travail en 1978, exception faite du MAS, MEQ et de la fonction publique québécoise pour lesquels l'information désirée est facilement accessible sans questionnaire. Le taux de réponses élevé (près de 98\%) est fort encourageant quant à la représentativité des répondants et la nature des questions ne devraient pas poser de problèmes de biais systématique. Tel ne serait pas le cas si l'on ajoutait des questions relatives aux causes des arrêts et aux conditions de reprise du travail. Par contre, de telles informations jumelées à celles déjà recueillies par l'enquête feraient faire un bond fantastique à la compréhension du phénomène.

Voilà pour l'ajout incontestablement prometteur du rapport. Autres changements: le calcul des jours-personnes perdus a subi deux légères modifications, l'une de forme désexisant l'ancienne appellation de «jourshommes perdus", l'autre, de fond tenant compte du départ de travailleurs entre le début et la fin du conflit; le nombre de jours ouvrables, qui entrent dans le calcul du pourcentage de temps de travail perdu est passé de 259 à 252 afin de refléter davantage le nombre réel de jours fériés intégrés dans la plupart des conventions collectives. Comme le font remarquer les auteurs, la comparabilité d'avec les années antérieures en souffre quelque peu, aussi y aurait-il avantage a ajuster les données pour 1976 et 1977. Enfin, en plus du tableau II (indices globaux) qui permet une lecture comparative d'avec les années précédentes, les auteurs dans leur présentation du tableau III font également une telle comparaison. Comme nous l'avons déjà suggéré, il serait intéressant pour le lecteur de disposer d'information similaire pour d'autres tableaux, ou encore chaque tableau pourrait inclure un indice de variation annuelle.

\section{Jacques MERCIER}

Université Laval

Essentials of Personnal Management, par Mitchel S. Novit, Englewood Cliffs, N.J., Prentice-Hall Inc., 1979, 243 pp.

Personnel: The Management of Human Ressources, par Stephen P. Robbins, Englewood Cliffs, N.J., Prentice Hall Inc., 1978, 393 pp.

En rédigeant ces deux ouvrages, les auteurs ont voulu fournir aux étudiants de premier cycle universitaire, un instrument pédagogique utilisable au cours d'un trimestre d'enseignement. Les sujets traités sont identiques à ceux qu'on retrouve dans les 